\title{
Perfectionistic Profiles Among Elite Athletes and Differences in Their Motivational Orientations
}

\author{
Daniel F. Gucciardi, ${ }^{1}$ John Mahoney, ${ }^{1}$ Geoffrey Jalleh, ${ }^{2}$ \\ Robert J. Donovan, ${ }^{2}$ and Jarred Parkes ${ }^{1}$ \\ ${ }^{1}$ University of Queensland; ${ }^{2}$ Curtin University
}

\begin{abstract}
Although there is an emerging body of research that has examined perfectionistic clusters in the general population, few studies have explored such profiles in athlete samples. The purposes of this research were to explore perfectionistic profiles within a sample of elite athletes and the differences between them on key motivational variables. A sample of 423 elite athletes (179 males, 244 females) aged between 14 and 66 years $(M=25.64 ; S D=8.57)$ from a variety of team (e.g., rowing, hockey, baseball, rugby) and individual sports (e.g., cycling, athletics, triathlon, gymnastics) completed a multisection questionnaire including measures of sport perfectionism, motivation regulation, achievement goals, and fear of failure. Cluster analyses revealed the existence of three perfectionism profiles, namely, nonperfectionists, maladaptive perfectionists, and adaptive perfectionists. Subsequent analyses generally supported the robustness of these perfectionism profiles in terms of differential motivational orientations (achievement goals, fear of failure, and motivation regulation) in hypothesized directions. Overall, the differences in motivational orientations between the three clusters supported a categorical conceptualization of perfectionism.
\end{abstract}

Keywords: categorical conceptualization, achievement goals, fear of failure, cluster analysis, person-centered analyses

Regardless of the theoretical framework, it is generally agreed that perfectionism is a multidimensional construct involving both intra- and interpersonal aspects that is characterized by a tendency to strive for exceedingly high standards of performance (e.g., Flett \& Hewitt, 2002; Stoeber \& Otto, 2006). Within sport contexts, some have argued that perfectionism is an adaptive quality that helps athletes reach their potential (e.g., Gould, Dieffenbach, \& Moffett, 2002), whereas others have highlighted its maladaptive nature for achievement pursuits (e.g., Flett \& Hewitt, 2005). However, given that perfectionism is a multidimensional

Daniel F. Gucciardi, John Mahoney, and Jarred Parkes are with the School of Human Movement Studies, University of Queensland, Brisbane, QLD, Australia. Geoffrey Jalleh and Robert J. Donovan are with the Centre for Behavioural Research in Cancer Control, Curtin University, Perth, WA, Australia. 
construct, its functional nature appears more complex in that some perfectionistic facets are associated with adaptive outcomes and processes, while others are related to maladaptive behaviors and consequences (Enns \& Cox, 2002; Stoeber \& Otto, 2006). As perfectionism has been associated with both functional/healthy and dysfunctional/unhealthy psychological, affective, and behavioral correlates among competitive athletes (e.g., Gould et al., 2002; Hill \& Appleton, 2011; Hill, Hall, Appleton, \& Murray, 2010; Stoeber, Uphill, \& Hotham, 2009), it is of no surprise that perfectionism remains an important topic for future study.

Two prominent frameworks have guided much of the research on perfectionism. Frost, Marten, Lahart, and Rosenblate (1990) proposed that there are six primary facets of perfectionism: personal standards (the tendency to set high standards for oneself and place great importance on such standards); concern over mistakes (the tendency to view mistakes as failure that inevitably will lead to the loss of respect from others); organization (the desire and pursuit of orderliness); parental expectations (the tendency to perceive parents as holding high standards and being overly critical of oneself); parental criticism (the belief that parents critically evaluate one's actions); and doubts about actions (the lingering worry that tasks are never satisfactorily completed). In contrast, Hewitt and Flett (1991) posited that there are three primary facets of perfectionism: self-oriented perfectionism (the setting of excessively high standards for oneself accompanied by the tendency to be highly self-evaluative and self-critical); other-oriented perfectionism (the tendency to set unrealistic standards for others and be highly evaluative and critical of their actions); and socially prescribed perfectionism (the belief that others hold excessively high standards for your behavior and that the acceptance of others is contingent upon attaining those high standards). More recently, however, Stoeber and Otto (2006) presented a conceptual integration of these two approaches in which they argued that two primary dimensions of perfectionism can be revealed. The dimension of perfectionistic strivings represents a commitment to exceptionally high standards and encompasses facets of perfectionism that are typically considered normal, adaptive, and healthy (e.g., high personal standards). In contrast, the dimension of perfectionistic concerns represents a tendency to be overly critical in evaluations of one's behavior and encompasses facets of perfectionism that are typically considered abnormal, maladaptive, and unhealthy (e.g., concern over mistakes, doubts about actions, socially prescribed perfectionism). In addition, Stoeber and Otto argued that these two perfectionism dimensions could be employed to differentiate three types or kinds of perfectionists: healthy perfectionists typically have high levels of perfectionistic strivings with low levels of perfectionistic concerns; unhealthy perfectionists have high levels of both perfectionistic strivings and perfectionistic concerns; and nonperfectionists have low levels of perfectionistic strivings (Stoeber \& Otto, 2006).

Researchers have typically adopted one of two approaches to understanding the nature of perfectionism - namely, a dimensional approach and a categorical approach-which essentially view this construct as differing in degrees (i.e., a quantitative difference) or in kinds (i.e., a qualitative difference) (Stoeber \& Otto, 2006). Within the dimensional approach, which involves a quantitative difference in the degree or amount of perfectionistic facets one possess, perfectionism is best understood as comprising a continuum between low, moderate, and high levels of perfectionism. The common approach for researchers adopting the dimensional 
approach is to combine the different facets of perfectionism into the two aforementioned overarching dimensions (Stoeber \& Otto, 2006) and adopt "variablecentered analyses" (e.g., correlation, regression, structural equation modeling) to ascertain their functional significance with hypothesized antecedents, correlates, and outcomes. Research involving nonathlete participants has indicated that the two dimensions of perfectionism show differential patterns of relationships in hypothesized directions. Specifically, perfectionistic strivings are typically related to positive characteristics, processes, and outcomes such as positive affect, active coping styles, and life satisfaction, whereas perfectionistic concerns are largely associated with negative characteristics, processes, and outcomes such as neuroticism, negative affect, and suicide ideation (for a review, see Stoeber \& Otto, 2006). Research with athlete populations has provided additional support for the differential patterns of relationships of these two dimensions of perfectionism with related outcomes, processes, and characteristics such as achievement goals (e.g., Stoeber, Stoll, Salmi, \& Tiikkaja, 2009), performance (e.g., Stoeber, Uphill, et al., 2009), and burnout (e.g., Hill et al., 2010). In contrast, within the categorical approach, in which qualitative differences are said to exist in the facets of perfectionism one possess, perfectionism is best understood as differing in the types or kinds of perfectionists (i.e., adaptive perfectionists, maladaptive perfectionists, nonperfectionists). There is a considerable body of evidence from both sport (e.g., Gotwals, 2011; Martinent \& Ferrand, 2006; Sapieja, Dunn, \& Holt, 2011) and nonsport contexts (e.g., Chan, 2009; Hanchon, 2010; Parker, 1997; Rice \& Ashby, 2007; Rice \& Mirzadeh, 2000) that has provided support for a categorical conceptualization of perfectionism using "person-centered analyses" (e.g., cluster analysis), although rare exceptions do exist that have supported a dimensional view of perfectionism (e.g., Vallance, Dunn, \& Causgrove Dunn, 2006).

Due to the domination of variable-centered analyses (e.g., correlations, regression) when investigating perfectionism in sport, person-centered approaches (e.g., cluster analysis) have typically been neglected (e.g., Gotwals, 2011; Sapieja et al., 2011). The scarcity of person-centered research is concerning as an inherent assumption within variable-centered approach is that the population is homogenous with respect to the variable(s) of interest (Bergman \& Trost, 2006). Variable-centered methods, which essentially tell us that two or more variables are statistically related, do not say anything about the individual as they treat individuals as "replaceable, randomly selected data carriers" (Von Eye \& Bogat, 2006, p. 392). Person-centered approaches, on the other hand, identify groupings of people whose patterns of scores across perfectionism dimensions are homogenous within a group, but heterogeneous across groups (Stoeber \& Otto, 2006). Such an approach has the ability to highlight different individual profiles and provide a deeper understanding of perfectionism at the individual level. In particular, person-centered approaches can be used to report unique and unexpected subgroups of multidimensional constructs and the response pattern of individuals (Von Eye \& Bogat, 2006). Such conceptual insights are achieved by assessing facets of perfectionism simultaneously as opposed to independently. In addition, person-centered analyses allow greater conclusions to be drawn about perfectionism profiles that can better guide practical implications, as the individual is the central concern rather than the variables (Bergman \& Trost, 2006).

Person-centered approaches to understanding perfectionism in sport have not been completely neglected, however. Martinent and Ferrand (2006), for example, 
revealed the existence of three types of perfectionists (nonperfectionists, adaptive perfectionists, maladaptive perfectionists) among a sample of 166 competitive athletes using cluster analysis. Both the Sport Multidimensional Perfectionism Scale (Sport-MPS; Dunn, Causgrove Dunn, Gotwals, Vallance, Craft, \& Syrotuik, 2006; Dunn, Causgrove Dunn, \& Syrotuik, 2002) and Hewitt and Flett's (1991) Multidimensional Perfectionism Scale were employed by Martinent and Ferrand to identify unique clusters of perfectionists. Subsequent research that cluster analyzed a sample of 194 male youth soccer players' responses to the Sport Multidimensional Perfectionism Scale-2 (Gotwals \& Dunn, 2009) revealed support for this tripartite categorical conceptualization of perfectionism (Sapieja et al., 2011). More recently, Gotwals (2011) replicated the methodological approach of Sapieja et al. among a sample of 117 varsity athletes and extended the tripartite conceptualization to include two groups of unhealthy perfectionists. Specifically, while both groups of unhealthy perfectionists reported a tendency to set high personal standards and experience enhanced concerns over mistakes, one group perceived high expectations and criticisms from their parents (i.e., parent-oriented unhealthy), whereas the other group reported high levels of dissatisfaction or uncertainty with their training preparation for competition (i.e., doubt-oriented unhealthy). Only one study to date has supported a dimensional conceptualization of perfectionism when using a person-centered approach. Vallance et al. (2006) cluster analyzed a sample of 229 male youth ice hockey players' responses to the Sport-MPS and revealed support for the existence of three clusters corresponding to low, moderate, and high levels of each of the four perfectionism facets.

While the aforementioned person-centered research has important implications for understanding perfectionism in sport, the methodologies employed are not without limitation. In particular, the focus on either male adolescent athletes (Sapieja et al., 2011; Vallance et al., 2006) or small samples of competitive adult athletes (Gotwals, 2011; Martinent \& Ferrand, 2006) limits our confidence in the extent to which these findings generalize to the wider athlete population. In addition, researchers have suggested that profiles of adult elite athletes are characterized solely by adaptive perfectionism (Gould et al., 2002). However, without empirical evidence to support such claims, it is unreasonable to discount the existence of maladaptive perfectionism in such populations. Consequently, it remains unknown whether the categorical conceptualization of perfectionism exists among high-achieving, adult elite athletes and across age groups, gender, and sport types (i.e., individual versus team sports). Thus, as one of the most fundamental issues for progressing our understanding of the nature of perfectionism (Flett \& Hewitt, 2002), the primary aim of this study was to address these questions pertaining to the categorical or dimensional conceptualization dilemma.

Aligned with converging evidence from theory (Stoeber \& Otto, 2006) and research involving both athlete (e.g., Gotwals, 2011; Martinent \& Ferrand, 2006; Sapieja, Dunn, \& Holt, 2011) and nonathlete samples (e.g., Hanchon, 2010; Parker, 1997; Rice \& Ashby, 2007; Rice \& Mirzadeh, 2000), we expected to reveal support for a tripartite categorical conceptualization of perfectionism made up of nonperfectionists, adaptive perfectionists, and maladaptive perfectionists. To maintain consistency with previous research, we adopted the Sport-MPS (Dunn et al., 2002, Dunn, Causgrove Dunn, et al., 2006) as our central measure of perfectionism. Such domain-specific measures of perfectionism have been supported in research 
(e.g., Stoeber \& Stoeber, 2009), especially in sport (e.g., Dunn, Craft, Causgrove Dunn, \& Gotwals, 2011; Dunn, Gotwals, \& Causgrove Dunn, 2005). Recognizing the domain-specific nature of perfectionism, Dunn and colleagues developed the Sport-MPS as a means by which to measure perfectionism in sport settings (Dunn et al., 2002; Dunn, Causgrove Dunn, et al., 2006). The Sport-MPS encompasses four facets of perfectionism: three of these dimensions are grounded in Frost and colleagues' (1990) conceptual framework (personal standards, concerns over mistakes, and perceived parental pressure) and one extends the domain-general framework to sport settings in which the coach is recognized as a source of interpersonal or socially prescribed perfectionism (perceived coach pressure). Based on previous findings, we expected adaptive perfectionists to be characterized by high levels of personal standards and low levels of concerns over mistakes, perceived parent pressure, and perceived coach pressure; maladaptive perfectionists to be characterized by high levels of all four perfectionism facets; and nonperfectionists characterized by low levels of all four perfectionism facets.

\section{Motivational Correlates of Sport Perfectionism}

When adopting person-centered approaches, using additional variables other than those used to create subgroups is considered an efficient technique for validating the cluster solution and distinctiveness between groups (Aldenderfer \& Blashfield, 1984). Previous person-centered investigations of perfectionism in sport have carried out such procedures by examining differences between perfectionistic clusters on competitive anxiety intensity and direction (Martinent \& Ferrand, 2006), anger (Vallance et al., 2006), perceptions of parenting styles (Sapieja et al., 2011), and burnout (Gotwals, 2011). Aligned with this approach, the secondary aim of this study was to determine if perfectionistic profiles differed on other meaningful variables. In an attempt to extend the aforementioned literature, we considered both maladaptive and adaptive qualities that may provide clues as to the functional nature of the perfectionist profiles. Specifically, we considered independent but related motivational variables; namely achievement goals, extrinsic and intrinsic motivation, and fear of failure.

\section{Achievement Goals}

As striving for the attainment of very high performance standards is a key aspect of perfectionism (e.g., Flett \& Hewitt, 2002; Stoeber \& Otto, 2006), it seemed logical to examine differences in goal pursuits across the emergent perfectionistic profiles. Although different conceptualizations and operationalizations exist, we adopted Elliot and McGregor's (2001) $2 \times 2$ achievement goal framework in the current study: mastery approach (MAp), mastery avoidance (MAv), performance approach (PAp), and performance avoidance (PAv). Mastery approach goals motivate individuals to strive for the attainment of self/task-referenced competence, for example, striving to master a task. Those individuals motivated by MAv goals strive to avoid self/task-referenced incompetence, for example, avoiding doing a task worse than previous attempts. Those individuals motivated by PAp goals are focused on attaining normative competence, for example striving to do as well or better than others. Finally, those individuals motivated by PAv goals avoid normative 
incompetence, for example, they strive to avoid doing worse than others. A considerable body of research has supported the adaptive nature of approach goals (i.e., MAp, PAp) and the maladaptive nature of avoidance goals (i.e., MAv, PAv), with mastery approach goals being the most strongly related to adaptive outcomes and processes (for a review, see Roberts, Treasure, \& Conroy, 2007). Aligned with Slade and Owens's (1998) dual process model and Stoeber and Otto's (2006) common conceptual framework of perfectionism, as well as recent empirical research with athlete samples (e.g., Kaye, Conroy, \& Fifer, 2008; Stoeber, Stoll, Pescheck, \& Otto, 2008; Stoeber, Stoll, et al., 2009), we expected perfectionistic profiles to evidence differential levels of achievement goals. Specifically, adaptive perfectionists should evidence a more adaptive achievement goal profile (i.e., higher MAp and PAp, and lower PAv, MAv) than maladaptive perfectionists. With all four perfectionism facets expected to be low in the nonperfectionist cluster (Stoeber \& Otto, 2006), this group should evidence lower scores on all four achievement goals than both the maladaptive and adaptive perfectionist clusters.

\section{Fear of Failure}

The aversive achievement motive, fear of failure, was included in the current study, as it is considered a central regulatory feature of perfectionism (Blatt, 1995). Atkinson and Feather (1966) defined fear of failure as a "disposition to avoid failure and/ or capacity for experiencing shame or humiliation as a consequence of failure" ( $p$. 13). As a shame-based avoidance motive, individuals who have learned to associate failure with aversive consequences will typically perceive failure as threatening and experience fear and apprehension in evaluative situations (Conroy, Willow, \& Metzler, 2002). Conceptually, both fear of failure and aspects of perfectionism are concerned with the perceived critical nature of oneself or others and share a focus on avoiding incompetence. Thus, we expected fear of failure to be most strongly linked with the intra- (i.e., concerns over mistakes) and interpersonal (i.e., perceived parent pressure, perceived coach pressure) aspects of perfectionism captured by the SportMPS that relate to avoiding incompetence, but not with the intrapersonal aspect (i.e., personal standards) concerned with approaching high levels of performance. These conceptual expectations are also supported by recent empirical evidence derived from variable-centered research with physical activity students (Conroy, Kaye, \& Fifer, 2007; Kaye et al., 2008) and competitive athletes (Sagar \& Stoeber, 2009). Consequently, the maladaptive perfectionist cluster should report higher levels of fear of failure than both the adaptive perfectionist and nonperfectionist clusters, whereas no differences should exist between the adaptive perfectionist and nonperfectionist clusters.

\section{Motivation}

While perfectionism is defined, in part, by the setting of high standards, it also entails the motivational need to strive for and meet those standards (Hamachek, 1978). In terms of motivational regulations, it is important to distinguish intrinsic and extrinsic motivation, that is, whether individuals perceive their actions as autonomous and self-determined or as externally controlled and non-self-determined (Ryan \& Deci, 2000). Thus, we examined differences between the perfectionistic 
clusters on both intrinsic and extrinsic motivation in the current study. Aligned with self-determination theory (Deci \& Ryan, 2000) and the dual-process model of perfectionism (Slade \& Owens, 1998), we expected intrinsic motivation to be most strongly linked with the personal standards component of perfectionism, which is associated with approaching achievement via self-referenced criteria and the desiring or striving for perfection for internally based reasons (e.g., enjoyment, satisfaction of accomplishment). In contrast, extrinsic motivation should be most strongly related to those intra- (i.e., concerns over mistakes) and interpersonal (i.e., perceived parent pressure, perceived coach pressure) aspects of perfectionism that are concerned with avoiding blame and negative evaluations from others. These conceptual expectations are largely supported by recent empirical evidence derived from variable-centered research both within sport (e.g., Gaudreau \& Antl, 2008; McArdle \& Duda, 2004) and nonsport contexts (e.g., Gaudreau \& Thompson, 2010; Stoeber, Feast, \& Hayward, 2009). Therefore, the nonperfectionists should report lower levels of intrinsic and extrinsic motivation than both the adaptive and maladaptive perfectionists; no differences in intrinsic motivation should exist between the adaptive and maladaptive perfectionists; and the maladaptive perfectionists should report higher levels of extrinsic motivation than the adaptive perfectionists.

\section{Purposes of the Present Study}

In summary, the purposes of this paper were to explore the number and types of perfectionistic profiles in elite athlete populations, and what the implications of these distinct perfectionistic profiles may be for motivational orientations. As we focused solely on elite athletes, this study also provided an opportunity to empirically examine the contention that adult elite athletes are characterized solely by adaptive perfectionistic profiles (e.g., Gould et al., 2002). In line with previous theory (e.g., Stoeber \& Otto, 2006) and research (e.g., Gotwals, 2011; Martinent \& Ferrand, 2006; Sapieja et al., 2011), we expected to reveal support for a tripartite categorical conceptualization of perfectionism made up of nonperfectionists, adaptive perfectionists, and maladaptive perfectionists. In addition, we expected the adaptive perfectionist cluster to report a more adaptive motivational orientation (i.e., higher MAp and PAp, and lower PAv and MAv goals; lower fear of failure; higher intrinsic motivation, lower extrinsic motivation) than the maladaptive cluster.

\section{Methods}

\section{Participants}

The sample consisted of 423 elite athletes (179 males, 244 females) aged between 14 and $66(M=25.64 ; S D=8.57)$. The sports represented included a variety of team (e.g., rowing, hockey, baseball, rugby) and individual sports (e.g., cycling, athletics, triathlon, gymnastics); three participants did not report their sport. Athletes' highest level of competition included the Olympics $(n=120)$ and World Championships $(n$ $=303$ ), with 175 having attained an international title, and 195 having attained a national title. The majority of participants $(88 \%)$ were highly experienced having competed in their sport for at least five or more years. 


\section{Instrumentation}

Sport Multidimensional Perfectionism Scale (Sport-MPS; Dunn et al., 2002). The Sport-MPS contains 30 items that measure four facets of perfectionism in sport: personal standards (seven items, e.g., "I have extremely high goals for myself in my sport"); concern over mistakes (eight items, e.g., "If I play well but only make one obvious mistake in the entire game, I still feel disappointed with my performance"); perceived parental pressure (nine items, e.g., "In competition, I never feel like I can quite meet my parents' expectations"); and perceived coach pressure (six items, e.g., "Only outstanding performance in competition is good enough for my coach"). Participants responded to each item on a 5-point scale ranging from strongly disagree (1) to strongly agree (5). Factor analytical research supports the four-factor structure of the Sport-MPS (see Dunn, Causgrove Dunn, et al., 2006; Dunn et al., 2002), with adequate levels of internal reliability consistently observed for each of the four perfectionism subscales (e.g., Gotwals \& Dunn, 2009; Gotwals, Dunn, Causgrove Dunn, \& Gamache, 2010; Vallance et al., 2006). External validity evidence also exists in terms of links between athletes' perfectionistic tendencies and achievement goals (Dunn et al., 2002), anger (Dunn, Gotwals, Causgrove Dunn, \& Syrotuik, 2006; Vallance et al., 2006), competitive anxiety (Martinent, Ferrand, Guillet, \& Gautheur, 2010), and athletic performance (Stoeber, Uphill, et al., 2009).

Achievement Goals Questionnaire-Sport (AGQ-S; Conroy, Elliot, \& Hofer, 2003). The 12-item AGQ-S measures the degree to which participants endorse four different achievement goals in their sport: mastery approach (e.g., "It is important to me to perform as well as I possibly can"), mastery avoidance (e.g., "I worry that I may not perform as well as I possibly can"), performance approach (e.g., "It is important to me to do well compared to others"), and performance avoidance (e.g., "My goal is to avoid performing worse than everyone else"). Participants responded to each item on a 7-point scale ranging from not at all like me (1) to completely like me (7). Factor analytical research supports the four-factor structure of the AGQ-S (Adie, Duda, \& Ntoumanis, 2008; Conroy et al., 2003; Nien \& Duda, 2008), with adequate levels of internal reliability consistently observed for each of the four achievement goals (e.g., Conroy et al., 2003). External validity evidence also exists in terms of links between athletes' goal orientations and perceived competence, both extrinsic and intrinsic motivation (Nien \& Duda, 2008), mental toughness (Gucciardi, 2010), appraisals of threat and challenge, self-esteem, and affect (Adie et al., 2008).

Performance Failure Appraisal Inventory (PFAl; Conroy et al., 2002). This 5-item short form derived from the PFAI measures participants' perceived degree of fear regarding failure in sport (e.g., "When I am failing, it upsets my "plan" for the future"). Participants responded to each item on a 5-point scale ranging from do not believe at all (-2) to believe $100 \%$ of the time (2). Factor analytical research supports the unidimensional structure of the PFAI (Conroy et al., 2002; Nien \& Duda, 2008), with adequate levels of internal reliability consistently observed for the global fear of failure construct (e.g., Conroy, Coatsworth, \& Kaye, 2007; Conroy et al., 2002; Nien \& Duda, 2008). In addition, the short-form is highly correlated with the long-form measure ( $r=.92$; Conroy et al., 2002). External validity evidence also exists in terms of links between fear of failure and motivation, anxiety (Conroy, 
Coatsworth, et al., 2007), achievement goals (Conroy et al., 2003; Nien \& Duda, 2008), sport anxiety, perceived competence, and hope (Conroy et al., 2002).

Sport Motivation Scale-6 (SMS-6; Mallett, Kawabata, Newcombe, OteroForero, \& Jackson, 2007). The SMS-6 is a 24-item inventory that measures six motivational orientations consistent with the framework of self-determination theory (Deci \& Ryan, 1985) that are intended to identify athletes' perceived reasons for participating in sport. Only the four external regulation ("Because it allows me to be well regarded by people that I know") and four intrinsic motivation ("For the excitement I feel when I am really involved in the activity") items were employed in this study. Participants responded to each item on a 7-point scale ranging from does not correspond at all (1) to corresponds exactly (7). Factor analytical research supports the six-factor structure of the SMS-6 (Lonsdale, Hodge, \& Rose, 2008; Mallett et al., 2007), with adequate levels of internal reliability consistently observed for each of the six motivation subscales (e.g., Gucciardi, 2010; Mallett et al., 2007). External validity evidence also exists in terms of links between athletes' motivational tendencies and dispositional flow (Lonsdale et al., 2008; Mallett et al., 2007), mental toughness (Gucciardi, 2010), and burnout (Lonsdale et al., 2008).

\section{Procedure}

Institutional ethics approval was obtained before the commencement of this study. An information sheet describing the aims and procedures of the project as well as the research proposal was sent to relevant personnel (e.g., director, sport science manager) at each Australian Institute of Sport/Sport Academy and other national sporting bodies (e.g., Hockey Australia, Australian Water Polo). Questionnaire packages were then couriered to athletes and included a reply-paid envelope; instructions were provided within the questionnaire package. The survey aimed to collect a comprehensive psychological evaluation of elite athletes and therefore contained a number of other psychological constructs not reported here. The study variables of interest to the current study appeared in the following order in the larger survey package: SMS-6, AGQ-S, Sport-MPS, and PFAI. Participants were informed that the study examined psychological variables of performance in sport, honesty in responses was essential, participation was voluntary, and responses would remain confidential. A 33\% response rate was achieved in the current study and, although low, fell within recommended research guidelines (Baruch \& Holtom, 2008).

\section{Data Analysis}

All analyses were conducted using SPSS 18.0. Following an examination of missing values, data were screened (i.e., univariate and multivariate normality and outliers, homogeneity of variance-covariance matrices) and internal reliability estimates (i.e., Cronbach's alpha) were assessed for each construct before conducting the main analyses. Descriptive statistics (i.e., means, standard deviations, bivariate correlations) were calculated to provide a description of the sample.

First, we performed a series of one-way MANOVAs to ascertain whether perfectionism varied as a function of gender, sport type, and age. In terms of sport type, participants were categorized as either a team $(n=191)$ or individual $(n=$ 229) sport athlete; three athletes did not report their sport. Chronological age was 
divided into three groups: aged less than 20 years $(n=106)$, aged $20-30$ years $(n$ $=227$ ), and aged 31 years and above $(n=90)$. An ANOVA with Scheffe's post hoc test for comparisons of means was employed to examine a significant multivariate effect, as it corrects the alpha level to guard against inflation of Type I error rates as a result of multiple comparisons (Tabachnick \& Fidell, 2007).

Second, cluster analysis was performed to classify athletes into groups with similar patterns of scores across the four perfectionism subscales. The first stage involved a hierarchical cluster analysis using Ward's linkage method with the squared Euclidian distance measure to provide guidance as to the number of clusters represented in the data. Ward's hierarchical method was chosen because it reduces the within cluster differences found in other methods (Aldenderfer \& Blashfield, 1984). We used standardized $z$ scores of the four perfectionism subscales in the clustering analyses (Hair, Black, Babin, \& Anderson, 2010). In the second stage, $k$-means (nonhierarchical) cluster analysis using simple Euclidean distance as the similarity measure was conducted, specifying the solution identified using Ward's method. To explore the stability of the final solution, we implemented the two-thirds random sample reclustering procedure, discriminant function analysis (Gore, 2000; Hair et al., 2010), and the double-split cross-validation procedure (Breckenridge, 2000).

Third, a MANOVA using cluster group as the independent variable and the other motivational variables (i.e., achievement goals, motivation, fear of failure) as the dependent variables was performed. An alpha level of .05 was employed with partial eta squared $\left(\eta^{2}\right)$ providing an index of effect size. An ANOVA with Scheffe's post hoc test for comparisons of means was employed to examine a significant multivariate effect, with a Bonferroni correction applied to guard against inflation of Type I error rates as a result of multiple comparisons.

\section{Results}

\section{Descriptive Statistics, Reliability, and Correlations}

The complete dataset was examined for missing values before the main analyses. When less than $5 \%$ of data points are randomly missing, almost any procedure for handling missing values yields similar results (Tabachnick \& Fidell, 2007). Of the 423 participants, only 90 athletes returned a questionnaire package with any missing data. In each case at least one and no more than seven data points were missing (total points missing $=121$ or $.53 \%$ of the total dataset). As the level of missing data were unrelated to any of the demographic (i.e., age, gender, sport type) or study variables (i.e., perfectionism, achievement goals, fear of failure, motivation), we implemented the expectation maximization method for data imputation. The expectation-maximization method has been shown to be an accurate technique for replacing missing data of this kind (Graham, 2009).

Descriptive statistics, reliability estimates, and correlations among all study variables using the total sample are presented in Table 1. All constructs demonstrated good reliability (i.e., Cronbach's $\alpha \geq .70$; Nunnally \& Bernstein, 1994). Data screening procedures did not identify any variables as non-normal (i.e., skewness/ kurtosis $>2$ ) or participants as multivariate outliers (i.e., using a $p<.001$ criterion for Mahalanobis $D^{2}$ ), although there were five univariate outliers $(z> \pm 3.0)$. Because 


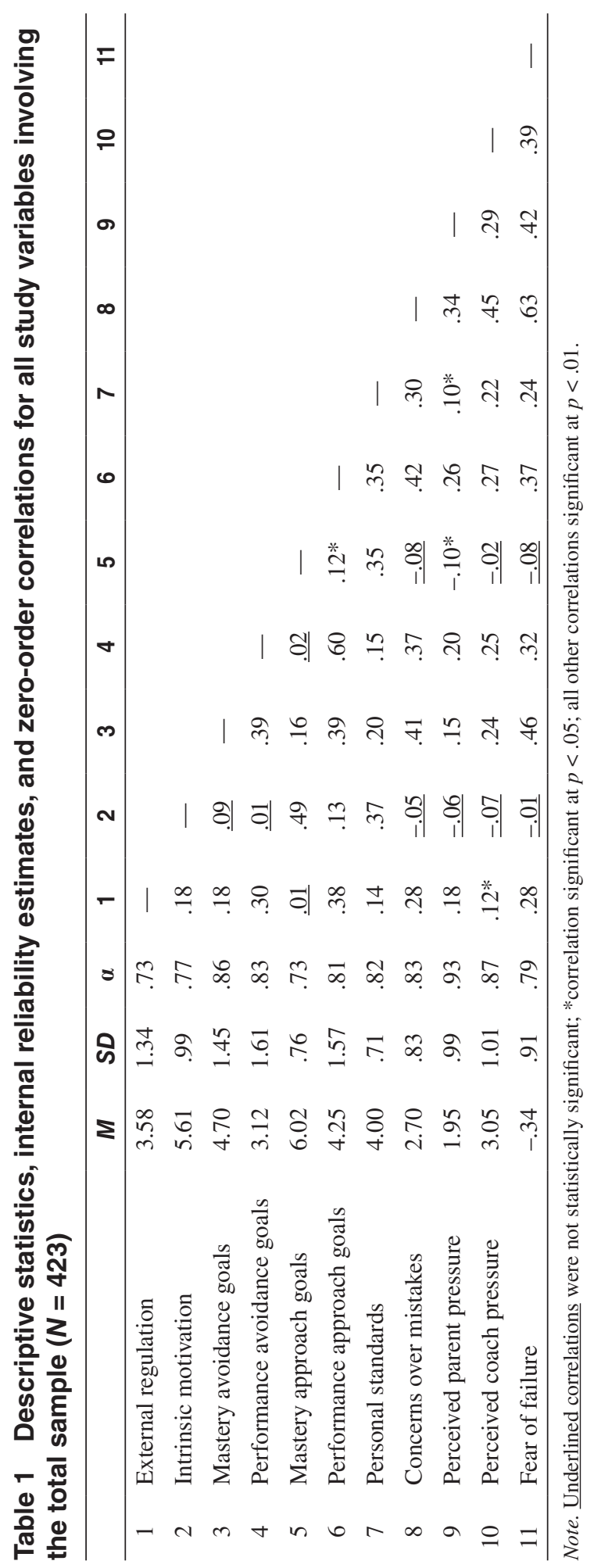


outliers can have a significant impact on the outcomes of a cluster analysis (Hair et al., 2010), separate analyses were run both with and without the outliers. However, all cases were retained in the cluster analysis as the results were comparable for both analyses. Low-to-moderate correlations existed between the motivational variables, thereby supporting a simultaneous analysis using MANOVA.

\section{Differences in Perfectionism Across Gender, Sport Type, and Age}

The equality of both error and covariance matrices were tested for each MANOVA using Levene's test and Box's $M$ test, respectively. Analyses revealed the error variances of the dependent variables to be equal across all groups (i.e., Levene's test $p$ $>$.05). Tests of the equality of covariance matrices among the four perfectionism subscales revealed significant differences between gender (Box's $M=19.51, p=$ .04 ) and sport type (Box's $M=23.94, p=.01$ ) but not chronological age (Box's $M=30.61, p=.07)$. However, homogeneity of covariance can be assumed unless Box's $M$ is significant at $p<.001$ (Tabachnick \& Fidell, 2007).

A series of one-way MANOVAs were performed to ascertain whether perfectionism varied as a function of gender, sport type, and age. There were no significant differences between male and female athletes on the multivariate combination of the four perfectionism components, Wilks's $\Lambda=.64, F(4,418)=.64, p=.63$. However, MANOVAs showed a significant multivariate effect of age, Wilks's $\Lambda=.93$, $F(4,417)=5.02, p=.001, \eta^{2} \mathrm{p}=.03$, and sport type, Wilks's $\Lambda=.95, F(4,415)=$ $5.02, p=.001, \eta_{\mathrm{p}}^{2}=.05$, on the four perfectionism subscales. With regard to sport type, follow-up ANOVAs revealed a significant effect of sport type on concern over mistakes, $F(1,418)=5.94, p=.015, \eta_{\mathrm{p}}^{2}=.01$, and coach pressure, $F(1,418)=7.21$, $p=.008, \eta^{2}{ }_{\mathrm{p}}=.02$, but not personal standards, $F(1,418)=.01, p=.904, \eta_{\mathrm{p}}^{2}=.00$, and parent pressure, $F(1,418)=3.10, p=.079, \eta_{\mathrm{p}}^{2}=.01$. Specifically, individual sport athletes reported lower levels of concern over mistakes $(M=2.61, S D=.86)$ and perceived coach pressure $(M=2.92, S D=1.07)$ than team sport athletes $(M$ $=2.87, S D=.94 ; M=3.19, S D=.93$, respectively). With regard to age, follow-up ANOVAs revealed a significant effect of sport type on perceived parent pressure, $F$ $(2,420)=4.95, p=.008, \eta_{\mathrm{p}}^{2}=.02$, and coach pressure, $F(2,420)=4.64, p=.01, \eta^{2} \mathrm{p}$ $=.02$, but not personal standards, $F(2,420)=.01, p=.904, \eta_{\mathrm{p}}^{2}=.00$, and concern over mistakes, $F(2,420)=3.10, p=.079, \eta^{2}{ }_{p}=.01$. Scheffe post hoc analyses revealed that athletes aged under 20 years reported significantly $(p=.006)$ higher levels of perceived parent pressure $(M=2.18, S D=1.12)$ than athletes aged over 30 years $(M=1.75, S D=.96)$. In contrast, athletes aged between 20 and 30 years reported significantly $(p=.009)$ higher levels of perceived coach pressure $(M=$ $3.18, S D=1.97)$ than those athletes aged under 20 years $(M=2.82, S D=1.03)$. Noting the minimal differences in perfectionism both in terms of the number (i.e., only four significant mean differences) and magnitude (i.e., small effect sizes) of these differences, we decided to combine the entire sample for the main analyses.

\section{Perfectionistic Profiles}

The agglomeration coefficient and dendrograms generated from Ward's hierarchical method identified three solutions (two, three, and four clusters) as candidates to 
be considered in the nonhierarchical analysis. Thus, we used the cluster centroids from the hierarchical Ward analysis as the initial seed points in subsequent nonhierarchical $k$-means analyses. A three-cluster solution was deemed the best fit according to empirical (i.e., balance of amount of variance explained to number of participants changing clusters from the hierarchical to nonhierarchical analysis, similar representation of participants in each cluster across gender, age, and sport type groups) and conceptual (i.e., consistency with previous empirical research and theory) considerations. An overview of the distribution of athletes within each cluster according to gender, sport type, and age group is detailed in Table 2. No significant differences were found in the distribution of these demographic variables across the clusters.

Results of the stability analyses indicated that $92 \%$ of participants retained their original cluster membership using a two-thirds random sample (Gore, 2000), whereas $95 \%$ of participants were correctly classified using a discriminant function analysis. We also randomly generated two subsamples from the total pool of participants (Sample A = 223; Sample B = 200) and conducted the full two-stage procedure (i.e., Ward followed by $k$-means) with each subsample to assess the stability of the three cluster solution. Similarly, we performed this two-stage procedure within subsamples of the participants according to gender, sport type, and age groups. In each subsample assessment, the level of agreement between the cluster solutions produced by the Ward and $k$-means techniques was assessed by means of a Cohen's kappa ( $\kappa)$ (cf. Breckenridge, 2000). With a value of .60 considered acceptable (Breckenridge, 2000), the results revealed that there was good agreement between the two stages for both Sample A $(\kappa=.77)$ and Sample B $(\kappa=.74)$. Acceptable levels of agreement between the Ward and $k$-means solutions were also observed for gender $(\kappa=.74$ and .67$)$, age groups $(\kappa=.79, .65$, and .72$)$, and sport type ( $\kappa=.68$ and .76$)$. Collectively, these cross-validation techniques increased our confidence that the three-cluster solution has a certain degree of generality and stability because a similar solution emerged across samples drawn from the same population (Aldenderfer \& Blashfield, 1984).

We next employed MANOVA to examine mean levels of perfectionism subscales across the three clusters. A MANOVA showed a significant multivariate effect of cluster membership on the four perfectionism subscales, Wilks's $\Lambda=.18$, $F(4,417)=135.95, p<.001, \eta^{2} \mathrm{p}=.56$. The results of the follow-up ANOVAs are detailed in Table 3. Specifically, Cluster 1 reported significantly lower levels of personal standards and concerns over mistakes than Cluster 2 and Cluster 3; Cluster 1 reported significantly lower levels of perceived parent pressure and perceived coach pressure than Cluster 2; and Cluster 2 reported significantly higher levels of concerns over mistakes, perceived parent pressure, and perceived coach pressure than Cluster 3.

In addition to the aforementioned statistical analyses of mean levels of perfectionism, we examined the interpretability of the three-cluster solution by inspecting both relative (i.e., standardized scores; $z$ scores) and absolute (i.e., raw scores; actual subscale scores) scores. Aligned with previous research employing cluster analyses (e.g., Harwood, Cumming, \& Fletcher, 2004; Hodge, Allen, \& Smellie, 2008), we employed a standardized score of \pm 50 to indicate high and low levels of each facet, with scores in between (i.e., +.50 to -.50$)$ to indicate moderate levels. The first cluster ( $n=131,31 \%$ of total sample) was labeled non-perfectionists, 


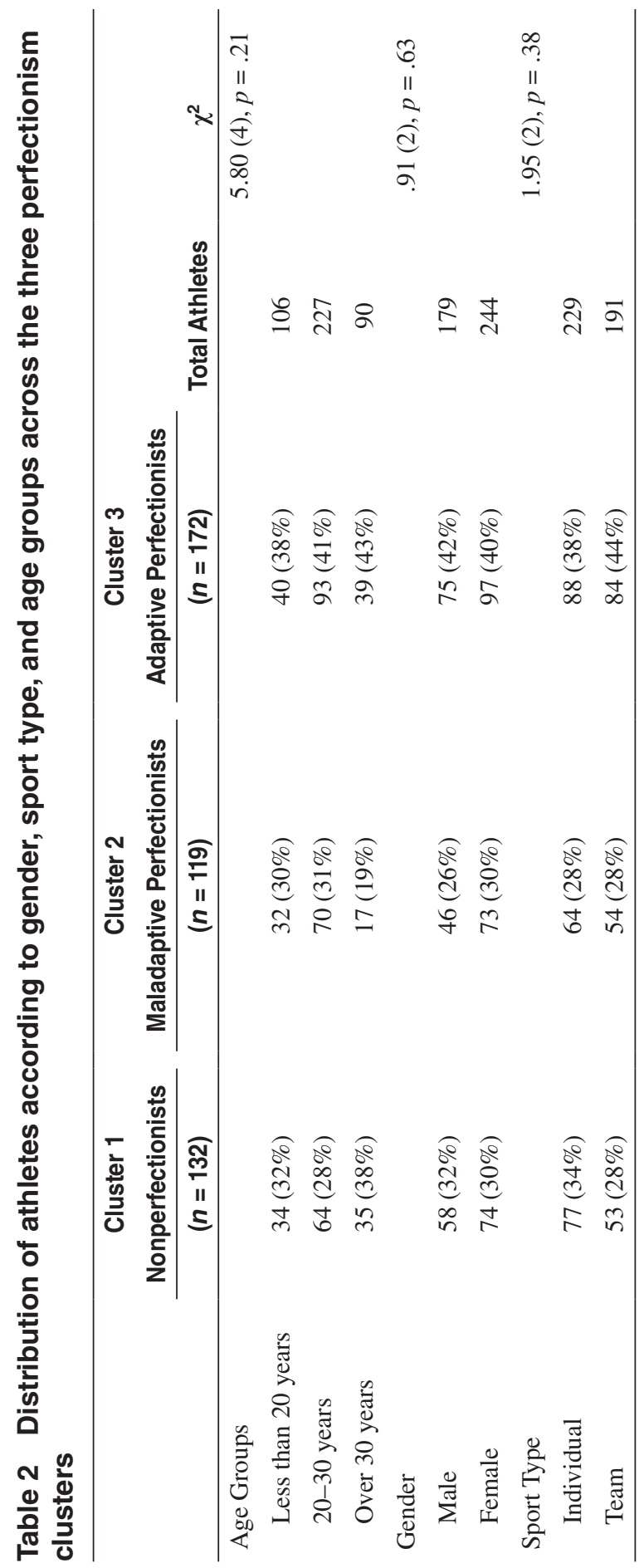




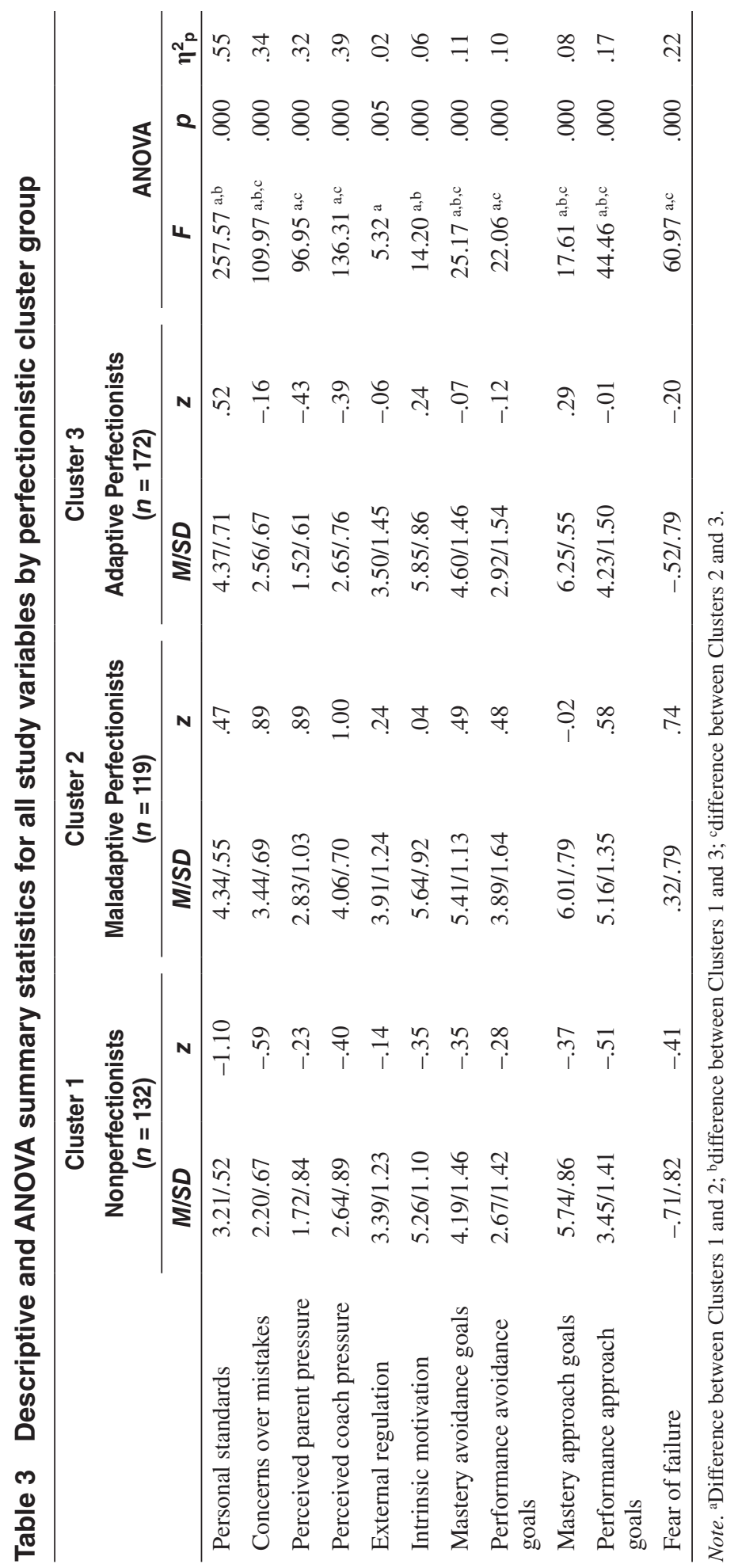


as participants reported low levels of both personal standards and concerns over mistakes, and moderate levels of perceived parent pressure and perceived coach pressure. The second cluster ( $n=119,28 \%$ of total sample) was labeled maladaptive perfectionists, as participants reported high levels of concerns over mistakes, perceived parent pressure, and perceived coach pressure, with personal standards just below the .50 cutoff for a high score. Finally, the third cluster $(n=172,41 \%$ of total sample) was labeled adaptive perfectionists, as participants reported high levels of personal standards combined with low levels of concerns over mistakes, and moderate levels of perceived parent pressure and perceived coach pressure.

\section{Differences in Motivational Variables Between Perfectionistic Profiles}

A significant multivariate effect for perfectionism cluster on the motivational variables, Wilks's $\Lambda=.63, F(7,414)=15.34, p<.001, \eta_{p}^{2}=.21$, was evidenced with all components contributing to the significant multivariate effect (see Table 3 ). As is detailed in Table 3, nonperfectionists reported lower levels of extrinsic motivation $(p=.009)$, intrinsic motivation $(p=.006)$, MAv $(p<.001)$, PAv $(p<.001)$, MAp $(p=.018)$, PAp $(p<.001)$, and fear of failure $(p<.001)$ than maladaptive perfectionists; nonperfectionists reported lower levels of intrinsic motivation $(p<$ $.001)$, MAv $(p=.038)$, MAp $(p<.001)$, and PAp goals $(p<.001)$ than adaptive perfectionists; maladaptive perfectionists reported higher levels of MAv $(p<.001)$, PAv $(p<.001)$, PAp goals $(p<.001)$, and fear of failure $(p<.001)$ than adaptive perfectionists; and maladaptive perfectionists reported lower levels of MAp goals $(p=.025)$ than adaptive perfectionists.

\section{Discussion}

The purposes of this study were to explore the profiles of perfectionism within a heterogeneous elite athlete sample, and examine differences in the motivational orientations of the emergent clusters. Consistent with theory (Stoeber \& Otto, 2006) and empirical evidence (e.g., Gotwals, 2011; Martinent \& Ferrand, 2006; Sapieja et al., 2011), we revealed support for a tripartite categorical conceptualization of perfectionism encompassing groups of nonperfectionists, adaptive perfectionists, and maladaptive perfectionists. Our expectations regarding the motivational orientations of these profiles were largely supported. Adaptive perfectionists demonstrated significantly higher levels of MAp goals, and lower levels of fear of failure and PAv, MAv, and PAp goals than the maladaptive perfectionists. In addition, the nonperfectionists reported significantly lower levels on all seven motivational orientations than maladaptive perfectionists, and lower levels of intrinsic motivation, MAp, and PAp goals than the adaptive perfectionists. Contrary to expectations, no significant differences in extrinsic motivation existed between adaptive perfectionists and both nonperfectionists and maladaptive perfectionists; no significant differences emerged between adaptive perfectionists and nonperfectionists on PAv goals; and the difference between maladaptive and adaptive perfectionists on PAp goals was in the opposite direction. 


\section{Perfectionist Profiles in Elite Athletes}

Theory (e.g., Slade \& Owens, 1998; Stoeber \& Otto, 2006) and research (e.g., Martinent \& Ferrand, 2006; Sapieja et al., 2011) has supported the notion that perfectionism might best be viewed as a construct involving both adaptive/healthy and maladaptive/unhealthy processes and outcomes. This distinction is particularly relevant in the world of elite sport where setting high standards and striving for excellence and perfection are often considered common and desirable among those athletes who reach and perform at the highest level (Gould et al., 2002). Nevertheless, the issue of whether perfectionism is best conceptualized from a dimensional or categorical perspective continues to spark debate among theorists and researchers (e.g., Flett \& Hewitt, 2002). Adding to this body of knowledge, the current findings have provided additional support for a tripartite categorical conceptualization of perfectionism encompassing groups of nonperfectionists, adaptive perfectionists, and maladaptive perfectionists.

Aligned with the common conceptual model of perfectionism (Stoeber \& Otto, 2006), both the adaptive and maladaptive perfectionists reported similarly high levels of personal standards. However, the maladaptive perfectionists were also typified by high levels of concerns over mistakes, perceived parent pressure, and perceived coach pressure, whereas the adaptive perfectionists were accompanied by low-to-moderate levels of these three perfectionism facets. These findings are consistent with previous person-centered research that has identified perfectionism clusters of the type found in the current study among athlete samples (e.g., Gotwals, 2011; Martinent \& Ferrand, 2006; Sapieja et al., 2011). The presence of higher levels of intra- (i.e., concerns over mistakes) and interpersonal (i.e., perceived parent pressure, perceived coach pressure) aspects of perfectionism likely drive maladaptive perfectionists to avoid incompetence or negative consequences, whereas lower levels of these additional perfectionistic facets allow an individual to strive for or approach competence or positive consequences (Slade \& Owens, 1998). Further, statistically significant difference between the maladaptive perfectionists and adaptive perfectionists pertained to concerns over mistakes, perceived parental pressure, and perceived coach pressure, thereby underscoring the importance of these facets in qualitatively distinguishing these two types of perfectionists. Thus, it may be argued that having high standards for performance is not problematic unless combined with overly critical self-evaluations of behavior of performance (Frost et al., 1990; Stoeber \& Otto, 2006), and perceptions of high expectations and criticisms from significant others (i.e., coaches, parents).

The emergence of a nonperfectionist cluster is also consistent with previous research (Gotwals, 2011; Martinent \& Ferrand, 2006; Sapieja et al., 2011) and theory (Stoeber \& Otto, 2006). Consistent with expectations, the nonperfectionist group reported significantly lower levels of personal standards than both the maladaptive and adaptive perfectionist clusters. In addition, the nonperfectionist group reported significantly lower levels of the three perfectionism facets captured by the Sport-MPS, which align with Stoeber and Otto's perfectionistic concerns dimension, than the maladaptive perfectionists. Similar distinctions were evidenced between the adaptive and maladaptive clusters. Specifically, concern over mistakes, perceived parental pressure, and perceived coach pressure were all significantly 
different between groups such that maladaptive perfectionists were higher on all three facets. These findings underscore the importance of these three facets when differentiating categorical clusters of perfectionists.

Our confidence in the parsimony and stability of the three-cluster solution was enhanced by the fact that a similar tripartite profile of perfectionism emerged across age groups, gender, and sport types (i.e., individual versus team sports) as well as different methodological approaches (i.e., two-thirds random sample reclustering, discriminant function analysis; Gore, 2000; Hair et al., 2010). While variable-centered analyses revealed differences (albeit small in magnitude) in four perfectionism facets among age groups and sport types, additional analyses revealed that, overall, athletes from different age groups, gender, and sport types were proportionally represented among the four clusters. Data pertaining to the stability of the cluster solution and proportional representation of athletes appears to have contradicted empirical evidence that perfectionism decreases with age in nonathlete samples (e.g., Landa \& Bybee, 2007; Stoeber \& Stoeber, 2009).

Consistent with a categorical conceptualization of perfectionism, however, it may well be that different facets of perfectionism rather than an overall level of this construct are what decrease or increase with age. Indeed, our analyses indicated that athletes aged 20 years or younger reported higher levels of perceived parental pressure than athletes aged 30 years and above. In addition, athletes aged 20-30 years reported significantly higher levels of perceived coach pressure than athletes aged 20 years or younger. These findings appear consistent with the contention that parents are an important source of performance-related information during the early years but become less influential as athletes get older and are more dependent on the feedback of key actors (e.g., coaches) in the sport context (Dunn, Gotwals, \& Causgrove Dunn, 2005; Gotwals, Dunn, \& Wayment, 2003). We are not aware of any research to date that has examined differences in perfectionism between individual and team sport athletes thereby this aspect of the current study represented an important contribution to the sport perfectionism literature that requires replication and extension in future research. To our knowledge, the current study represented one of the most comprehensive examinations of an emergent cluster solution across subgroups of an athlete sample to date. Nevertheless, additional research is required to ascertain the robustness of the current findings across independent samples of athletes.

\section{Differences in Motivational Orientations}

We attempted to further our understanding of the functional nature of perfectionism by examining differences between athletes with differing perfectionistic profiles on both maladaptive and adaptive motivational orientations. It was envisaged that integrating dispositional and achievement motivational perspectives would enhance theoretical conceptualizations of perfectionism in sport that have typically been obtained using variable-centered approaches. Collectively, the categorical interpretation of the profiles of perfectionism was generally supported by the differing motivational orientations reported by the emergent clusters.

Consistent with theoretical (e.g., Slade \& Owens, 1998; Stoeber \& Otto, 2006) and empirical expectations (e.g., Stoeber et al., 2008), the adaptive perfectionists reported significantly higher levels of MAp goals, and lower levels of PAv and MAv 
goals than the maladaptive perfectionists. However, contrary to the tenets outlined in the dual process theory of perfectionism (Slade \& Owens, 1998) and empirical evidence generated from variable-centered research (e.g., Stoeber et al., 2008), the adaptive cluster also reported lower levels of PAp goals than the maladaptive cluster. While our correlational data were aligned with these expectations, the current findings highlight that when individual level data are considered (i.e., clusters) adaptive perfectionists can be further defined by the desire to attain internally derived standards of competence. In contrast, maladaptive perfectionists appear to be defined by the desire to avoid incompetence relative external and internal standards, as well as attain externally derived standards of competence. Unlike previous assertions regarding the variation between perfectionistic strivings and concerns (e.g., Kaye et al., 2008), the current findings underscore the importance of considering both definitions of competence (i.e., mastery vs. performance) and the valence of their focal outcome (i.e., avoiding competence vs. approaching competence) when distinguishing between maladaptive and adaptive perfectionists.

Beyond their varying achievement goal profiles, the adaptive perfectionists also reported significantly lower levels of fear of failure than the maladaptive perfectionists. This finding is consistent with theoretical (e.g., Slade \& Owens, 1998) and empirical observations (e.g., Kaye et al., 2008; Sagar \& Stoeber, 2009). While the correlational data indicated a positive association between all four perfectionism components and global fear of failure (cf. Kaye et al., 2008), the differences in mean levels across the adaptive and maladaptive perfectionists supported the centrality of intra- (i.e., concerns over mistakes) and interpersonal (i.e., perceived parent pressure, perceived coach pressure) aspects of perfectionism that relate to avoiding incompetence which distinguish these two types of perfectionists. That a significant difference in fear of failure emerged between the maladaptive and nonperfectionists (i.e., distinguished by all four perfectionism facets), but not between adaptive and nonperfectionists (i.e., distinguished by personal standards and concerns over mistakes), provided further support for the idea that having high standards for performance may not problematic unless combined with indicators of overly critical self-evaluations of behavior of performance (Frost et al., 1990; Stoeber \& Otto, 2006). Nevertheless, it is important that future person-centered research examines differences in the five fears of failure between the tripartite perfectionist clusters, as we only considered its global factor.

Mixed findings were evidenced in relation to differences in intrinsic and extrinsic motivation between the three perfectionism clusters. With regard to intrinsic motivation, no significant differences existed between the maladaptive and adaptive perfectionists, whereas the nonperfectionists reported lower levels than both the maladaptive and adaptive perfectionists. These findings are consistent with theoretical expectations (e.g., Deci \& Ryan, 2000; Slade \& Owens, 1998; Stoeber \& Otto, 2006) in which intrinsic motivation is considered to be most strongly linked with the personal standards facet, as it represents an internally motivated form of perfectionism (e.g., approach achievement via self-referenced criteria, strive for perfection for internally-based reasons). In contrast, no significant differences in extrinsic motivation existed between the maladaptive and adaptive perfectionists, and between the adaptive and nonperfectionists. As extrinsic motivation should be most strongly related to those intra- (i.e., concerns over mistakes) and interpersonal (i.e., perceived parent pressure, perceived coach pressure) aspects of perfectionism 
that are concerned with avoiding blame and negative evaluations from others (e.g., Gaudreau \& Antl, 2008; Gaudreau \& Thompson, 2010; McArdle \& Duda, 2004), the finding with regard to adaptive and nonperfectionists is likely due to the lack of differences between these two clusters on the perceived parent and coach pressures. However, these three components of perfectionism were central to the distinction between maladaptive and adaptive perfectionists and are therefore inconsistent with these expectations. While these findings suggest that high personal standards may be associated with less adaptive forms of extrinsic motivation thereby casting some doubt as to the pure adaptive nature of this dimension (cf. Flett \& Hewitt, 2005), an inspection of the raw scores for each cluster indicated only moderate levels of the motivational drive. Indeed, elite athletes have reported using both intrinsic and extrinsic motivational processes (Mallett \& Hanrahan, 2004). Further research is required to ascertain the robustness of these findings.

\section{Strengths, Limitations, Future Research, and Conclusions}

The current research was strengthened by the use of a person-centered approach that overcame limitations of previous literature that had rarely considered the unique perfectionistic profiles of athletes. A person-centered approach allows greater conclusions to be drawn about perfectionism types and better guides practical implications as the individual is the central concern rather than the variables as in a variable-centered approach (Bergman \& Trost, 2006). The large sample of elite athletes encompassing a diverse representation of both Olympic (e.g., gymnastics, rowing, hockey) and non-Olympic sports (e.g., rugby league and union, ballet, Australian football) represented a second strength of the current study. Whereas previous research has sampled elite, adolescent athletes (Sapieja et al., 2011; Vallance et al., 2006) and competitive athletes (Gotwals, 2011; Martinent \& Ferrand, 2006), our focus on a representative sample of the sport population provided preliminary insight into the robustness of the perfectionistic profiles across individual and team sports, age groups, and gender. Finally, the inclusion of multiple indicators of both maladaptive and adaptive motivational orientations facilitated a more detailed analysis of the functional nature of the emergent profiles than has previously been reported in the sport literature (e.g., Gotwals, 2011; Martinent \& Ferrand, 2006; Sapieja et al., 2011).

Despite the strengths of our study, several limitations existed. Firstly, the research could be criticized for using the Sport-MPS as the only measure of perfectionism. As mentioned by Vallance et al. (2006), it is possible that the overall construct of perfectionism is underrepresented by the Sport-MPS because it does not include all of the dimensions of the Frost-MPS that it was originally modeled on. Gotwals and colleagues (Gotwals \& Dunn, 2009; Gotwals et al., 2010) have since established the validity of the Sport-MPS-2 that includes components of perfectionism not considered by our investigation, namely doubt about actions and organization (we collected data before the publication of the Sport-MPS-2). The use of such a measure may have identified other unique groups (cf. Gotwals, 2011) determined by the components of perfectionism than was possible from the four-component model used in our research and future research could improve on our methodology by utilizing the Sport-MPS-2. Secondly, future research would benefit from assessing the entire spectrum of motivation, as we focused only on 
two major components of this continuum (i.e., extrinsic and intrinsic motivation). Neglecting other important aspects of the motivational continuum (i.e., amotivation, introjected regulation, identified regulation, integrated regulation) limited our ability to provide a detailed interpretation of the findings within the overarching self-determination theory framework (e.g., see Mouratidis \& Michou, 2011; Stoeber, Feast, \& Hayward, 2009). Finally, owing to the cross-sectional nature of our study, we were unable to interpret causality from the findings. For example, we could not conclude whether the motivational variables assessed in the current study are a consequence or antecedent of the perfectionistic profiles reported. An understanding of the causal relationship of perfectionism could allow individuals concerned with the well-being of athletes to be better placed to address their concerns (e.g., fear of failure and performance avoidance). In addition, prospective studies would provide useful information as to the temporal stability of these profiles (e.g., over an entire competitive season), as there is some evidence to suggest that different facets of perfectionism (e.g., social prescribed perfectionism) decrease over time while others (e.g., self-oriented perfectionism) can remain relatively unchanged (e.g., Stoeber, Otto, \& Dalbert, 2009).

In summary, the current study has contributed to the conceptualization of perfectionism in sport in several meaningful ways. Unlike previous person-centered research on perfectionism in sport (e.g., Gotwals, 2011; Martinent \& Ferrand, 2006; Sapieja et al., 2011), which has focused solely on male, adolescent or competitive, adult athletes, this study revealed support for a categorical conceptualization of perfectionism that is consistent with the common conceptual framework (Stoeber \& Otto, 2006) in a large sample of elite athletes. In addition, stability analyses generally supported the robustness of the tripartite conceptualization across age groups, gender, and sport type (i.e., individual vs. team sports). The significance of this tripartite categorical conceptualization of perfectionism was generally supported through differential motivational orientations of these clusters. Based on the current findings, it appears that achievement goals and the aversive achievement motive, fear of failure, but not intrinsic and extrinsic motivation, are central constructs to understanding the functional differences between types of perfectionistic clusters when individual level data are considered (i.e., clusters). More broadly, we hope to have demonstrated the usefulness of person-centered approaches as an important complement to the variable-centered research that dominates the field.

\section{Acknowledgments}

Gucciardi is supported by a University of Queensland Postdoctoral Research Fellowship. Appreciation is extended to two anonymous reviewers for the constructive feedback on earlier versions of this manuscript.

\section{References}

Adie, J.W., Duda, J.L., \& Ntoumanis, N. (2008). Achievement goals, competition appraisals, and the psychological and emotional welfare of sport participants. Journal of Sport \& Exercise Psychology, 30, 302-322.

Aldenderfer, M.S., \& Blashfield, R.K. (1984). Cluster analysis. Newbury Park, CA: Sage. Atkinson, J.W., \& Feather, N.T. (1966). A theory of achievement motivation. New York: Wiley. 
Baruch, Y., \& Holtom, B.C. (2008). Survey response rate levels and trends in organizational research. Human Relations, 61, 1139-1160.

Bergman, L.R., \& Trost, K. (2006). The person-oriented versus the variable-oriented approach: Are they complementary, opposites, or exploring different worlds? MerrillPalmer Quarterly, 52, 601-632.

Blatt, S.J. (1995). The destructiveness of perfectionism: Implications for the treatment of depression. The American Psychologist, 50, 1003-1020.

Breckenridge, J.N. (2000). Validating cluster analysis: Consistent replication and symmetry. Multivariate Behavioral Research, 35, 261-285.

Chan, D.W. (2009). Dimensionality and typology of perfectionism: The use of the Frost Multidimensional Perfectionism Scale with Chinese gifted students in Hong Kong. Gifted Child Quarterly, 53, 174-187.

Conroy, D.E., Coatsworth, J.D., \& Kaye, M.P. (2007). Consistency of fear of failure score meanings among 8- to 18-year-old female athletes. Educational and Psychological Measurement, 67, 300-310.

Conroy, D.E., Elliot, A.J., \& Hofer, S.M. (2003). A 2 x 2 Achievement Goals Questionnaire for Sport: Evidence for factorial invariance, temporal stability, and external validity. Journal of Sport \& Exercise Psychology, 25, 456-476.

Conroy, D.E., Kaye, M.P., \& Fifer, A.M. (2007). Cognitive links between fear of failure and perfectionism. Journal of Rational-Emotive \& Cognitive-Behavior Therapy, 25, 237-253.

Conroy, D.E., Willow, J.P., \& Metzler, J.N. (2002). Multidimensional fear of failure measurement: The Performance Failure Appraisal Inventory. Journal of Applied Sport Psychology, 14, 76-90.

Deci, E.L., \& Ryan, R.M. (1985). Intrinsic motivation and self-determination in human behaviour. New York: Plenum Press.

Deci, E.L., \& Ryan, R.M. (2000). The "What" and "Why" of goal pursuits: human needs and the self determination of behaviour. Psychological Inquiry, 11, 227-268.

Dunn, J.G.H., Causgrove Dunn, J.L., Gotwals, J.K., Vallance, J.K.H., Craft, J.M., \& Syrotuik, D.G. (2006). Establishing construct validity evidence for the Sport Multidimensional Perfectionism Scale. Psychology of Sport and Exercise, 7, 57-79.

Dunn, J.G.H., Causgrove Dunn, J., \& Syrotuik, D.G. (2002). Relationship between multidimensional perfectionism and goal orientation in sport. Journal of Sport \& Exercise Psychology, 24, 376-395.

Dunn, J.G.H., Gotwals, J.K., \& Causgrove Dunn, J. (2005). An examination of the domain specificity of perfectionism among intercollegiate student-athletes. Personality and Individual Differences, 38, 1439-1448.

Dunn, J.G.H., Gotwals, J.K., Causgrove Dunn, J., \& Syrotuik, D.G. (2006). Examining the relationship between perfectionism and trait anger in sport. International Journal of Sport and Exercise Psychology, 4, 7-24.

Elliot, A.J., \& McGregor, H.A. (2001). A 2 x 2 goal achievement framework. Journal of Personality and Social Psychology, 80, 501-519.

Enns, M.W., \& Cox, B.J. (2002). The nature and assessment of perfectionism: A critical analysis. In G.L. Flett \& P.L. Hewitt (Eds.), Perfectionism: Theory, research, and treatment (pp. 33-62). Washington, DC: American Psychological Association.

Flett, G.L., \& Hewitt, P.L. (2002). Perfectionism and maladjustment: An overview of theoretical, definitional, and treatment issues. In G.L. Flett \& P.L. Hewitt (Eds.), Perfectionism: Theory, research, and treatment (pp. 5-13). Washington, DC: American Psychological Association.

Flett, G.L., \& Hewitt, P.L. (2005). The perils of perfectionism in sport and exercise. Current Directions in Psychological Science, 14, 14-18.

Frost, R.O., Marten, P., Lahart, C., \& Rosenblate, R. (1990). The dimensions of perfectionism. Cognitive Therapy and Research, 14, 449-468. 
Gaudreau, P., \& Antl, S. (2008). Athletes' broad dimensions of dispositional perfectionism: Examining changes in life satisfaction and the mediating role of sport-related motivation and coping. Journal of Sport \& Exercise Psychology, 30, 356-382.

Gaudreau, P., \& Thompson, A. (2010). Testing a 2 x 2 model of dispositional perfectionism. Personality and Individual Differences, 48, 532-537.

Gore, P.A. (2000). Cluster analysis. In H. Tinsley \& S. Brown (Eds.), Handbook of applied multivariate statistics and mathematical modelling (pp. 298-321). San Diego, CA: Academic Press.

Gotwals, J.K. (2011).Perfectionism and burnout within intercollegiate sport: A personoriented approach. The Sport Psychologist, 25, 489-510.

Gotwals, J.K., \& Dunn, J.G.H. (2009). A multi-method multi-analytic approach to establishing internal construct validity evidence: The Sport Multidimensional Perfectionism Scale 2. Measurement in Physical Education and Exercise Science, 13, 71-92.

Gotwals, J.K., Dunn, J.G.H., Causgrove Dunn, J., \& Gamache, V. (2010). Establishing validity evidence for the Sport Multidimensional Perfectionism Scale-2 in intercollegiate sport. Psychology of Sport and Exercise, 11, 423-432.

Gotwals, J.K., Dunn, J.G.H., \& Wayment, H.A. (2003). An examination of perfectionism and self-esteem in intercollegiate athletes. Journal of Sport Behavior, 26, 17-37.

Gould, D., Dieffenbach, K., \& Moffett, A. (2002). Psychological characteristics and their development in Olympic champions. Journal of Applied Sport Psychology, 14, 172-204.

Graham, J.W. (2009). Missing data analysis: Making it work in the real world. Annual Review of Psychology, 60, 549-576.

Gucciardi, D.F. (2010). Mental toughness profiles and their relations with achievement goals and sport motivation in adolescent Australian footballers. Journal of Sports Sciences, 28, 615-625.

Hair, J.F., Black, W.C., Babin, B.J., \& Anderson, R.E. (2010). Multivariate data analysis (7th ed.). Englewood Cliffs, NJ: Prentice-Hall.

Hamachek, D.E. (1978). Psychodynamics of normal and neurotic perfectionism. Psychology (Savannah, Ga.), 15, 27-33.

Hanchon, T.A. (2010). The relations between perfectionism and achievement goals. Personality and Individual Differences, 49, 885-890.

Harwood, C., Cumming, J., \& Fletcher, D. (2004). Motivational profiles and psychological skills use within elite youth sport. Journal of Applied Sport Psychology, 16, 318-332.

Hewitt, P.L., \& Flett, G.L. (1991). Perfectionism in the self and social contexts: Conceptualization, assessment, and association with psychopathology. Journal of Personality and Social Psychology, 60, 456-470.

Hill, A.P., \& Appleton, P.R. (2011). The predictive ability of the frequency of perfectionistic cognitions, self-oriented perfectionism, and social prescribed perfectionism in relation to symptoms of burnout in youth rugby players. Journal of Sports Sciences, 29, 695-703.

Hill, A.P., Hall, H.K., Appleton, P.R., \& Murray, J.J. (2010). Perfectionism and burnout in canoe polo and kayak slalom athletes: The mediating influence of validation and growth seeking. The Sport Psychologist, 24, 16-34.

Hodge, K., Allen, J.B., \& Smellie, L. (2008). Motivation in Masters sport: Achievement and social goals. Psychology of Sport and Exercise, 9, 157-176.

Kaye, M.P., Conroy, D.E., \& Fifer, A.M. (2008). Individual differences in incompetence avoidance. Journal of Sport \& Exercise Psychology, 30, 110-132.

Landa, C.E., \& Bybee, J.A. (2007). Adaptive elements of aging: Self-image discrepancy, perfectionism, and eating problems. Developmental Psychology, 43, 83-93.

Lonsdale, C., Hodge, K., \& Rose, E.A. (2008). The Behavioural Regulation in Sport Questionnaire (BRSQ): Instrument development and initial validity evidence. Journal of Sport \& Exercise Psychology, 30, 323-355.

Mallett, C.J., \& Hanrahan, S.J. (2004). Elite athletes: Why does the 'fire' burn so brightly? Psychology of Sport and Exercise, 5, 183-200. 
Mallett, C., Kawabata, M., Newcombe, P., Otero-Forero, A., \& Jackson, S. (2007). Sport motivation scale-6 (SMS-6): A revised six-factor sport motivation scale. Psychology of Sport and Exercise, 8, 600-614.

Martinent, G., \& Ferrand, C. (2006). A cluster analysis of perfectionism among competitive athletes. Psychological Reports, 99, 723-738.

Martinent, G., Ferrand, C., Guillet, E., \& Gautheur, S. (2010). Validation of the French version of the Competitive State Anxiety Inventory-2 (CSAI-2) including frequency and direction scales. Psychology of Sport and Exercise, 11, 51-57.

McArdle, S., \& Duda, J.L. (2004). Exploring social-contextual correlates of perfectionism in adolescents: A multivariate perspective. Cognitive Therapy and Research, 28, 765-788.

Mouratidis, A., \& Michou, A. (2011). Perfectionism, self-determined motivation, and coping among adolescent athletes. Psychology of Sport and Exercise, 12, 355-367.

Nien, C.L., \& Duda, J.L. (2008). Antecedents and consequences of approach and avoidance achievement goals: A test of gender invariance. Psychology of Sport and Exercise, 9, 352-372.

Nunnally, J., \& Bernstein, I. (1994). Psychometric theory. New York: McGraw-Hill.

Parker, W.D. (1997). An empirical typology of perfectionism in academically talented children. American Educational Research Journal, 34, 545-562.

Rice, K.G., \& Ashby, J.S. (2007). An efficient method for classifying perfectionists. Journal of Counseling Psychology, 54, 72-85.

Rice, K.G., \& Mirzadeh, S.A. (2000). Perfectionism, attachment, and adjustment. Journal of Counseling Psychology, 47, 238-250.

Roberts, G.C., Treasure, D.C., \& Conroy, D.E. (2007). Understanding the dynamic of motivation in sport and physical activity: An achievement goal interpretation. In G. Tenenbaum \& R.C. Eklund (Eds.), Handbook of sport psychology (3rd ed., pp. 3-30). Hoboken, NJ: Wiley.

Ryan, R.M., \& Deci, E.L. (2000). Self-determination theory and the facilitation of intrinsic motivation, social development, and well-being. The American Psychologist, 55, 68-78.

Sagar, S.S., \& Stoeber, J. (2009). Perfectionism, fear of failure, and affective responses to success and failure: The central role of rear of experiencing shame and embarrassment. Journal of Sport \& Exercise Psychology, 31, 602-627.

Sapieja, K.M., Dunn, J.G.H., \& Holt, N.L. (2011). Perfectionism and perceptions of parenting styles in male youth soccer. Journal of Sport \& Exercise Psychology, 33, 20-39.

Slade, P.D., \& Owens, R.G. (1998). A dual process model of perfectionism based on reinforcement theory. Behavior Modification, 22, 372-390.

Stoeber, J., Feast, A.R., \& Hayward, J.A. (2009). Self-orientated and socially prescribed perfectionism: Differential relationships with intrinsic and extrinsic motivation and test anxiety. Personality and Individual Differences, 47, 423-428.

Stoeber, J., \& Otto, K. (2006). Positive conceptions of perfectionism: Approaches, evidence, challenges. Personality and Social Psychology Review, 10, 295-319.

Stoeber, J., Otto, K., \& Dalbert, C. (2009). Perfectionism and the Big Five: Conscientiousness predicts longitudinal increases in self-oriented perfectionism. Personality and Individual Differences, 47, 363-368.

Stoeber, J., \& Stoeber, F.S. (2009). Domains of perfectionism: Prevalence and relationships with perfectionism, gender, age, and satisfaction with life. Personality and Individual Differences, 46, 530-535.

Stoeber, J., Stoll, O., Pescheck, E., \& Otto, K. (2008). Perfectionism and goal orientations in athletes: Relations with approach and avoidance orientations in mastery and performance goals. Psychology of Sport and Exercise, 9, 102-121.

Stoeber, J., Stoll, O., Salmi, O., \& Tiikkaja, J. (2009). Perfectionism and achievement goals in young Finnish ice-hockey players aspiring to make the Under-16 national team. Journal of Sports Sciences, 27, 85-94. 
Stoeber, J., Uphill, M.A., \& Hotham, S. (2009). Predicting race performance in triathlon: The role of perfectionism, achievement goals, and personal goal setting. Journal of Sport \& Exercise Psychology, 31, 211-245.

Tabachnick, B.S., \& Fidell, L.S. (2007). Using multivariate statistics (5th ed.). Needham Heights, MA: Allyn and Bacon.

Vallance, J.K.H., Dunn, J.G.H., \& Causgrove Dunn, J.L. (2006). Perfectionism, anger, and situation criticality in competitive youth ice hockey. Journal of Sport \& Exercise Psychology, 28, 383-406.

Von Eye, A., \& Bogat, G.A. (2006). Person orientation: Concepts, results, and development. Merrill-Palmer Quarterly, 52, 390-420.

Manuscript submitted: November 8, 2010

Revision accepted: December 31, 2011 